\title{
Heterogeneity and Subclassification of Barcelona Clinic Liver Cancer Stage B
}

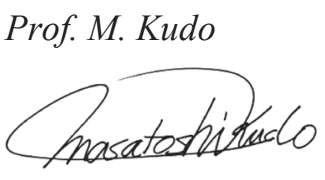

Editor Liver Cancer

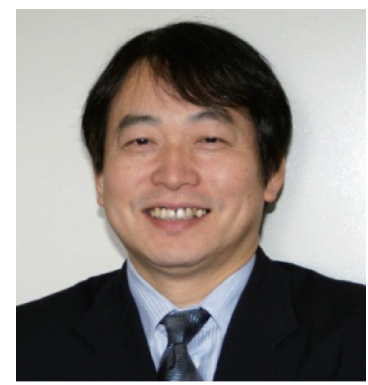

Intermediate-stage hepatocellular carcinoma ( $\mathrm{HCC}$ ) is defined as stage B in the Barcelona Clinic Liver Cancer (BCLC) staging system, the use of which is recommended by the American Association for the Study of Liver Disease [1] and the European Association for the Study of the Liver-European Organization for Research and Treatment of Cancer guidelines [2]. However, the concept of intermediate-stage HCC does not exist in the Japanese evidence-based guidelines [3], the Japanese consensus-based HCC treatment algorithm [4], or the Asian Pacific Association for the Study of the Liver guidelines [5]. A tentative definition of "intermediate stage" in the Japanese patient population suffering with HCC would probably include patients with Child-Pugh class A or B hepatic functional reserve and four or more tumors, as well as patients with a maximum tumor diameter of more than $3 \mathrm{~cm}$ who have no vascular invasion or extrahepatic spread. Transcatheter arterial chemoembolization (TACE) [6-10] is the only recommended treatment option for patients classified as intermediate stage under the BCLC classification. However, the intermediate stage includes a wide range of patient populations spanning from near-early-stage patients (treated with curative intent) to nearadvanced-stage patients, such as those with a Child-Pugh score of 9 (treated to preserve liver function or given best supportive care [BSC]). The subclassification of this heterogeneous intermediate-stage group and the design of treatment strategies specific for these substages have recently become topics of great interest.

\section{Heterogeneity of the Intermediate Stage}

BCLC stage B includes patients with Child-Pugh scores ranging from 5 to 9 , which results in a diverse group from the perspective of liver function. Moreover, the intermediate stage includes patients with as few as four tumors up to as many as 10 to $20+$ bilateral tumors as long as they have a performance status of 0 and do not have vascular invasion or extrahepatic spread. There is no upper limit of tumor size for the intermediate stage, which 
therefore includes all tumors larger than $3 \mathrm{~cm}$. As an extreme example, patients with three tumors that are just slightly larger than $3 \mathrm{~cm}$ and patients with one 5-6 cm tumors are both considered candidates for resection [11] as long as they have good hepatic functional reserve. In the case of patients with more than three tumors or a tumor that is more than $3 \mathrm{~cm}$, radiofrequency ablation (RFA) [12-14] is sometimes performed in combination with TACE if the tumor is not larger than approximately $6 \mathrm{~cm}$. In some cases, RFA is a good treatment option because large areas can be ablated using bipolar RFA equipment. Superselective lipiodol conventional TACE (CTACE) is typically performed as a treatment with curative intent. In cases with five or six tumors, a catheter is advanced superselectively to the vicinity of the tumors and lipiodol is injected, followed by a gelatin sponge as an embolic agent to induce partial hepatic infarction. The induced partial hepatic infarction can yield a complete response. Superselective cTACE can therefore serve as a highly advanced curative therapeutic technique. However, curative superselective cTACE is feasible only in tumors of a certain size and number, which explains why this procedure is rarely performed in countries other than Japan.

The use of TACE with drug-eluting beads mixed with chemotherapeutic agents (DEBTACE) has become more common in recent years.

This technique is most commonly used for large liver tumors and for palliative care or size reduction in patients who are not candidates for curative treatment. Both the decrease in hepatic functional reserve and the severity of postembolization syndrome after DEB-TACE are mild. Therefore, DEB-TACE is considered a better option than cTACE for treating large liver tumors over multiple sessions. Moreover, the use of lipiodol cTACE is not recommended in patients with a large number of bilateral tumors because it worsens the hepatic functional reserve in addition to being ineffective. These patients respond well to hepatic arterial infusion chemotherapy (HAIC). Because of the limited effect of DEB-TACE in patients with a large number of tumors, HAIC should be considered the first-line treatment for such patients. However, as liver function determines the prognosis of patients with a low tumor count and small tumors when hepatic functional reserve is relatively poor, the selected treatment must not reduce liver function if at all possible. For such patients, it is best to perform superselective lipiodol cTACE to minimize the reduction in hepatic functional reserve and to maximize tumor response. For patients with a Child-Pugh score of 5-7 who have numerous or large tumors, DEB-TACE or HAIC is recommended to achieve a good response while preserving liver function as much as possible. In patients with a poor hepatic functional reserve (i.e., Child-Pugh score 8-9), the treatment course is similar to that used for patients with Child-Pugh class C. In other words, although superselective cTACE and ablation are options if the tumor count and maximum tumor diameter are small, BSC or liver transplantation is typically indicated for these patients in accordance with the extended criteria. Another option is the administration of sorafenib (SOR) in patients with good liver function who have multiple bilateral tumors.

\section{Subclassification of the Intermediate Stage and Their Treatment Strategy}

Because of the heterogeneous nature of the intermediate-stage patient population, Bolondi et al. [15] proposed a subclassification for intermediate-stage HCC in 2012 (table 1). This subclassification is a very unique classification system that incorporates a new concept of joint consideration of the number of tumors and the maximum diameter according to the "beyond Milan" and "up-to-7" criteria. However, the assignment of patients to B1, B2, and B3 substages according to Child-Pugh score is complicated, and Child-Pugh scores of 5-6-7, 
Table 1. Subgrouping and treatment indications for patients with intermediate stage HCC [15]

\begin{tabular}{lllll}
\hline BCLC Substage & B1 & B2 & B3 & B4 \\
\hline $\begin{array}{l}\text { CPT score } \\
\begin{array}{l}\text { Beyond Milan } \\
\text { and within Up-to-7 }\end{array}\end{array}$ & $5-6-7$ & $5-6$ & 7 & $8-9$ \\
$\begin{array}{l}\text { ECOG (Tumor } \\
\text { Related) PS }\end{array}$ & IN & OUT & OUT & ANY \\
PVT & 0 & 0 & 0 & $0-1$ \\
$\begin{array}{l}\text { 1st option } \\
\text { Alternative }\end{array}$ & NO & NO & NO & NO \\
& TACE & TACE or TARE & BSC \\
& LT & SOR & Research trials & LT \\
& TACE + ablation & & TACE & \\
\hline
\end{tabular}

CPT=Child-Pugh-Turcotte; ECOG=Eastern Cooperative Oncology Group; PS=performance status; $\mathrm{PVT}=$ portal vein thrombosis; TARE=transarterial radioembolization; LT=liver transplantation.

Table 2. Treatment guidelines for patients with BCLC-B HCC in Japan (TAE Research Group of Japan) [17]

\begin{tabular}{llll}
\hline Subsubstage & Stage-B1 & Stage-B2 & Stage-B3 \\
\hline Liver Function and & CP score 5-6 & Other than & CP-9? \\
$\begin{array}{l}\text { Tumor status } \\
\text { Treatment option }\end{array}$ & $4-7 \mathrm{~cm} \mathrm{IN}$ & B1 and B3 & \\
& RFA & TACE & HAIC \\
Alternative & TACE (+RFA) & HAIC & BSC \\
& & SOR & \\
\hline
\end{tabular}

TAE=transarterial embolization; $\mathrm{CP}=$ Child-Pugh .

5-6, and 7 correspond to each substage. In addition, this subclassification does not differ from the original BCLC with respect to the first treatment option, in that TACE is typically recommended. However, it is superior for prognostication because the higher substages are associated with a worse prognosis after TACE. Another confusing issue is that portal vein thrombosis, a factor that was not originally included in the intermediate stage, is listed as "No" for all substages B1 through B4. It therefore seems unnecessary to include portal vein thrombosis as a factor. Moreover, although liver transplantation is suggested as an alternative treatment option for patients with a Child-Pugh score of 5,6 , or 7 , that suggestion is not very realistic. TACE and radioembolization are proposed as the first option for substage B2, although there is no established evidence for radioembolization. HAIC could be an alternative and it showed favorable results in the treatment of multiple bilateral tumors.

Yamakado et al. $[16,17]$ proposed a different subclassification system and treatment strategy (table 2). Kudo et al. proposed the Kinki criteria, which is a modification of Bolondi's classification that incorporates the ideas of classifying by Child-Pugh scores of 5-6-7 and 8-9 in addition to using the "beyond Milan" and "up-to-7" criteria from Bolondi's classification [15], resulting in the classification of patients into three subclasses by "IN" and "OUT" [18] (table 3). This system is simpler and easier to use than the original. Hepatic resection and ablation are also listed as treatment options for substage B1. The former is a good option if liver function is Child-Pugh A and there are one large tumor. The latter is an option if there are four to six small tumors. Ablation in combination with TACE is an option for large tumors of 4-6 cm because a large area can be ablated by this combined method. Moreover, in cases of four to six small tumors in the different subsegment of the liver, curative TACE can be per- 
Table 3. Subclassification and treatment strategies of intermediate-stage HCC (Kinki Criteria) [18]

\begin{tabular}{|c|c|c|c|c|}
\hline BCLC Substage & B1 & B2 & \multicolumn{2}{|c|}{ B3 } \\
\hline Child-Pugh Score & $5-7$ & $5-7$ & \multicolumn{2}{|c|}{8,9} \\
\hline \multirow{2}{*}{$\begin{array}{l}\text { Beyond Milan and } \\
\text { within up-to-7 }\end{array}$} & \multirow[t]{2}{*}{ IN } & \multirow[t]{2}{*}{ OUT } & \multicolumn{2}{|c|}{ ANY } \\
\hline & & & IN & OUT \\
\hline Subsubstage & & & B3-a & B3-b \\
\hline $\begin{array}{l}\text { Concept of Treatment } \\
\text { strategy }\end{array}$ & Curative intent & $\begin{array}{l}\text { Noncurative, } \\
\text { Palliative }\end{array}$ & $\begin{array}{l}\text { Curative intent if } \\
\text { within up-to-7 }\end{array}$ & $\begin{array}{l}\text { Palliative, } \\
\text { No treatment }\end{array}$ \\
\hline Treatment option & $\begin{array}{l}\text { Resection } \\
\text { Ablation } \\
\text { Superselective cTACE }\end{array}$ & $\begin{array}{l}\text { DEB-TACE } \\
\text { HAIC }^{\mathrm{b}} \\
\text { SOR }^{\mathrm{c}}\end{array}$ & $\begin{array}{l}\text { Transplantation } \\
\text { Ablation } \\
\text { Superselective cTACE }\end{array}$ & $\begin{array}{l}\text { HAIC } \\
\text { selective DEB-TACE }\end{array}$ \\
\hline Alternative & $\begin{array}{l}\text { DEB-TACE } \\
\text { (large, CP 7) } \\
\text { B-TACE }^{\text {d }}\end{array}$ & cTACE & $\begin{array}{l}\text { DEB-TACE } \\
\text { B-TACE }\end{array}$ & BSC \\
\hline
\end{tabular}

B-TACE=balloon-occluded transarterial chemoembolization.

${ }^{a}$ DEB-TACE is recommended for huge tumors that are $>6 \mathrm{~cm} .{ }^{\mathrm{b}}$ HAIC is recommended for multiple tumors $>6$ in total. ${ }^{c}$ SOR is recommended for patients with liver function of Child-Pugh score 5 and $6 .{ }^{d}$ B-TACE is recommended for fewer tumors.

formed by carefully targeting each tumor with superselective cTACE. If the superselective insertion of the catheter is not possible, DEB-TACE or balloon-occluded TACE [19] are additional options.

Repeated DEB-TACE was proposed for the treatment of large substage B2 liver tumors that are "beyond Milan," and it is a good treatment option for such large liver tumors. On the other hand, HAIC is effective for multiple HCCs that are "over up-to-7"; therefore, cTACE is not recommended for such tumors. Despite the use of cTACE in specific cases, it is not recommended because it reduces hepatic functional reserve. SOR treatment is another option when there are too many tumors to perform TACE. In conclusion, SOR should be considered as the initial treatment for patients with "over up-to-7" who are likely to quickly acquire refractoriness to cTACE and DEB-TACE [10, 20].

Substage B3 corresponds to Child-Pugh score 8 or 9 , and the standard treatment for this stage may be BSC according to Bolondi's subclassification. However, in patients who meet the "up-to-7" criteria, it is important to carefully treat each tumor individually by superselective cTACE or ablation, as this confers a survival benefit similar to that achieved in Child-Pugh C HCC [21]. Liver transplantation in accordance with the extended criteria or after downstaging should also be considered for "up-to-7" patients.

A validation study of the Kinki criteria showed good stratification of substages B1, B2, and B3 [22]. The survival curve for substage B1 was nearly identical to that for BCLC stage A, indicating that curative therapies such as superselective cTACE should be performed for this subgroup. Conversely, the survival curve for substage B3 overlapped with the curve for BCLC stage C. Therefore, since repeated cTACE in patients with substage B3 does not yield a better prognosis than that in patients with BCLC stage C, Arizumi et al. proposed HAIC or selective DEB-TACE for "beyond 7" patients with Child-Pugh score 8 or 9 (B3-b substage) and treatments that preserve liver function as much as possible, namely, superselective cTACE, or ablation for patients with B3-a substage. On the basis of these results, the authors noted that it is important to classify patients with BCLC stage B into these subcategories, and to consider both hepatic functional reserve (Child-Pugh score) and tumor factors (up-to-7 or beyond 7) when deciding upon a treatment strategy. 
Table 4. Subclassification of BCLC stage B HCC (Proposal by Korean Liver Cancer Study Group) B-TACE; Baloon occulded-TACE [23]

\begin{tabular}{l}
\hline Group definition \\
B1- Tumor size $<5 \mathrm{~cm}$ \\
B2- Tumor size $\geq 5 \mathrm{~cm}$ and Child-Pugh class A \\
B3- Tumor size $\geq 5 \mathrm{~cm}$ and Child-Pugh class B \\
\hline
\end{tabular}

Lee et al. [23] recently proposed a subclassification for BCLC stage B HCC based on registry data from the Korean Liver Cancer Study Group. In this subclassification, patients are classified by maximum tumor diameter (either $\geq 5 \mathrm{~cm}$ or $<5 \mathrm{~cm}$ ) and hepatic functional reserve (Child-Pugh A or B)(table 4). Subgroup B1 consists of patients with a maximum tumor diameter of less than $5 \mathrm{~cm}$ regardless of their Child-Pugh class, subgroup B2 consists of patients with Child-Pugh A and a maximum tumor diameter of more or equal to $5 \mathrm{~cm}$, and subgroup B3 consists of patients with Child-Pugh B and a maximum tumor diameter of more or equal to $5 \mathrm{~cm}$. The authors noted differences in the prognosis between these three groups. However, this classification is based on subcategories for prognostic factors of TACE, which differs from Bolondi's classification and the Kinki criteria.

\section{Conclusion}

This editorial has reviewed the heterogeneity of patients with intermediate-stage HCC and the significance of subclassification and treatment strategies. Four different subclassifications of intermediate-stage HCCs have been published to date. However, only Bolondi's subclassification, the Yamakado classification, and the Kinki criteria provide substages linked to proposed treatment strategies. These classifications need to be retrospectively validated in different cohorts and also their usefulness in the treatment of intermediate-stage HCCs needs to be prospectively validated.

\section{References}

1 Bruix J, Sherman M, American Association for the Study of Liver Diseases: Management of hepatocellular carcinoma: an update. Hepatology 2011;53:1020-1022.

2 Llovet JM, Ducreux M, et al European Association For The Study Of The Liver European Organisation For Research And Treatment Of Cancer: EASL-EORTC clinical practice guidelines: management of hepatocellular carcinoma. J Hepatol 2012;56:908-943.

3 Kokudo N, Hasegawa K, Akahane M, Igaki H, Izumi N, Ichida T, Uemoto S, Kaneko S, Kawasaki S, Ku Y, Kudo M, Kubo S, Takayama T, Tateishi R, Fukuda T, Matsui O, Matsuyama Y, Murakami T, Arii S, Okazaki M, Makuuchi M: Evidence-based clinical practice guidelines for hepatocellular carcinoma: The Japan Society of Hepatology 2013 update (3rd JSH-HCC guidelines). Hepatol Res, 2015;45 [epub ahead of print].

4 Kudo M, Matsui O, Izumi N, Iijima H, Kadoya M, Imai Y, Okusaka T, Miyayama S, Tsuchiya K, Ueshima K, Hiraoka A, Ikeda M, Ogasawara S, Yamashita T, Minami T, Yamakado K, Liver Cancer Study Group of Japan: JSH consensus-based clinical practice guideline for the management of hepatocellular carcinoma: 2014 update by the Liver Cancer Study Group of Japan. Liver Cancer 2014;3:458-468.

5 Omata M, Lesmana LA, Tateishi R, Chen PJ, Lin SM, Yoshida H, Kudo M, Lee JM, Choi BI, Poon RT, Shiina S, Cheng AL, Jia JD, Obi S, Han KH, Jafri W, Chow P, Lim SG, Chawla YK, Budihusodo U, Gani RA, Lesmana CR, Putranto TA, Liaw YF, Sarin SK: Asian Pacific Association for the Study of the Liver consensus recommendations on hepatocellular carcinoma. Hepatol Int 2010;4:439-474.

6 Raoul JL, Gilabert M, Piana G: How to define transarterial chemoembolization failure or refractoriness: a European perspective. Liver Cancer 2014;3:119-124.

7 Kudo M: Surveillance, diagnosis, treatment, and outcome of liver cancer in Japan. Liver Cancer 2015;4:3950. 
8 Kudo M: Locoregional therapy for hepatocellular carcinoma. Liver Cancer 2015;4:163-164.

9 Tsurusaki M, Murakami T: Surgical and locoregional therapy of hcc. Liver Cancer 2015;4:165-175.

10 Arizumi T, Ueshima K, Minami T, Kono M, Chishina H, Takita M, Kitai S, Inoue T, Yada N, Hagiwara S, Minami Y, Sakurai T, Nishida N, Kudo M: Effectiveness of sorafenib in patients with transcatheter arterial chemoembolization (TACE) refractory and intermediate-stage hepatocellular carcinoma. Liver Cancer 2015;4:253-262.

11 Ishizawa T, Kokudo N: The beginning of a new era of digestive surgery guided by fluorescence imaging. Liver Cancer 2014;3:6-8.

12 Teng W, Liu KW, Lin CC, Jeng WJ, Chen WT, Sheen IS, Lin CY, Lin SM: Insufficient ablative margin determined by early computed tomography may predict the recurrence of hepatocellular carcinoma after radiofrequency ablation. Liver Cancer 2015;4:26-38.

13 Kang TW, Rhim H: Recent advances in tumor ablation for hepatocellular carcinoma. Liver Cancer 2015;4:176-187.

14 Lencioni R, de Baere T, Martin RC, Nutting CW, Narayanan G: Image-guided ablation of malignant liver tumors: Recommendations for clinical validation of novel thermal and non-thermal technologies - a western perspective. Liver Cancer 2015;4:208-214.

15 Bolondi L, Burroughs A, Dufour JF, Galle PR, Mazzaferro V, Piscaglia F, Raoul JL, Sangro B: Heterogeneity of patients with intermediate (BCLC B) Hepatocellular Carcinoma: proposal for a subclassification to facilitate treatment decisions. Semin Liver Dis 2012;32:348-359.

16 Yamakado K, Miyayama S, Hirota S, Mizunuma K, Nakamura K, Inaba Y, Maeda H, Matsuo K, Nishida N, Aramaki T, Anai H, Koura S, Oikawa S, Watanabe K, Yasumoto T, Furuichi K, Yamaguchi M: Subgrouping of intermediate-stage (BCLC stage B) hepatocellular carcinoma based on tumor number and size and Child-Pugh grade correlated with prognosis after transarterial chemoembolization. Jpn J of Radiol 2014;32:260-265.

17 Yamakado K, Miyayama S, Hirota S, Mizunuma K, Nakamura K, Inaba Y, Yamamoto S, Matsuo K, Nishida N, Aramaki T, Anai H, Kora S, Oikawa S, Watanabe K, Yasumoto T, Furuichi K, Yamaguchi M: Prognosis of patients with intermediate-stage hepatocellular carcinomas based on the Child-Pugh score: Subclassifying the intermediate stage (Barcelona Clinic Liver Cancer stage B). Jpn J of Radiol 2014;32:644-649.

18 Kudo M, Arizumi T, Ueshima K, Sakurai T, Kitano M, Nishida N: Subclassification of BCLC B stage hepatocellular carcinoma and treatment strategies: Proposal of modified Bolondi's subclassification (Kinki criteria). Dig Dis 2015;33:751-758.

19 Irie T, Kuramochi M, Takahashi N: Dense accumulation of lipiodol emulsion in hepatocellular carcinoma nodule during selective balloon-occluded transarterial chemoembolization: measurement of balloonoccluded arterial stump pressure. Cardiovasc Intervent Radiol 2013;36:706-713.

20 Kudo M: Clinical practice guidelines for hepatocellular carcinoma differ between Japan, United States, and Europe. Liver Cancer 2015;4:85-95.

21 Kudo M, Osaki Y, Matsunaga T, Kasugai H, Oka H, Seki T: Hepatocellular carcinoma in Child-Pugh C cirrhosis: prognostic factors and survival benefit of nontransplant treatments. Dig Dis 2013;31:490-498.

22 Arizumi T, Ueshima K, Iwanishi M, Minami T, Chishina H, Kono M, Takita M, Kitai S, Inoue T, Yada N, Hagiwara S, Ida H, Minami Y, Sakurai T, Kitano M, Nishida N, Kudo M: Validation of a modified substaging system (Kinki criteria) for patients with intermediate-stage hepatocellular carcinoma. Oncology 2015;89(Suppl 2):47-52.

23 Lee S, Kim BK, Song K, Park JY, Ahn SH, Kim SU, Han KH, Kim DY, Korea Central Cancer Registry: Subclassification of Barcelona Clinic Liver Cancer B and C hepatocellular carcinoma: A cohort study of the multi-center registry database. J Gastroenterol Hepatol 2015 [Epub ahead of print]. 